Programa de Microbiología y Micología, Instituto de Ciencias Biomédicas (ICBM), Facultad de Medicina, Universidad de Chile, Santiago, Chile.

Conflictos de intereses: nada que declarar.

Recibido el 2 de septiembre de 2014, aceptado el 17 de diciembre de 2014

Correspondencia a:

Carlos G. Osorio Abarzúa Instituto de Ciencias Biomédicas (ICBM), Facultad de Medicina, Universidad de Chile, Santiago, Chile. gonosorio@med.uchile.cl

\section{Historia de los terrenos del Hospital Clínico y la Facultad de Medicina de la Universidad de Chile}

\author{
CARLOS G. OSORIO A.
}

\section{History of the University of Chile Faculty of Medicine and clinical hospital location}

The history of the location of the University of Chile Faculty of Medicine North Campus is derived from a farm of Pedro de Valdivia founder of the city of Santiago de la Nueva Extremadura and governor of the "Reyno de Chile". This work narrates succinctly the history of this particular location from the Spanish Conquest period to present days.

(Rev Med Chile 2015; 143: 252-256)

Key words: Chile; History of Medicine; Hospitals, teaching.
"Dónde están ahora todos aquellos señores y maestros....

En su vida parecían algo, ya no hay de ellos memoria. ¡Oh, cuan presto se pasa la gloria del mundo!

\section{Imitación de Cristo Tomás de Kempis}

\section{T} a historia del solar que ocupan actualmente el Campus Norte y el Hospital Clínico de la Facultad de Medicina de la Universidad de Chile es larga. Hay veces en que los avatares del devenir histórico se escapan de la simpleza y linealidad con que nuestro entendimiento pretende comprender el pasado, develando el estudio de los documentos originales una intrincada y compleja realidad, que destruye sin misericordia cualquier hipótesis preconcebida. Los resultados de este trabajo reflejan fielmente dicha aserción.

El solar de este estudio formó parte de una zona de la ciudad denominada "Chimba" (palabra derivada del idioma quechua que significa "del otro lado"), es decir, los terrenos aledaños a la ciudad que se localizaban al norte del río Mapocho. Recordemos que la ciudad de Santiago colonial se restringía en sentido norte-sur al espacio ubicado entre el cauce del río Mapocho y la actual avenida Libertador Bernardo O’Higgins
(Figura 1). Fue esta zona de la ciudad la elegida por el gobernador don Pedro de Valdivia para establecer su chácara (palabra derivada del idioma quechua que significa "mandíbula de asno" por el azadón que los incas usaban para cultivar sus campos; se simplificó posteriormente a "chacra"), posiblemente a causa de la excelente fertilidad de dichas tierras, pues se encontraban regadas por una intrincada red de acequias que provenían de la época prehispánica ${ }^{1,2}$. Los deslindes aproximados de la chacra del gobernador eran los siguientes: al norte el sector denominado el Salto de Araya (actual "Ciudad Empresarial”, en la comuna de Huechuraba); al sur el río Mapocho; al oriente la cadena de cerros del San Cristóbal y al poniente el "camino de Chile" (Figura 2). Se debe recordar que el camino real de Chile se denominaba Cañadilla en su extremo inicial (extremo sur) y camino real de Huechuraba en su extremo norte (también se le denominó "camino del Inca", pues probablemente fue utilizado por los incas para entrar a Santiago; actualmente corresponde en una gran proporción a la sección sur de la avenida Independencia).

\section{Chacra de doña Inés de Suárez en La Chimba}

El gobernador don Pedro de Valdivia, antes de dirigirse a una nueva campaña al sur en 1550, 


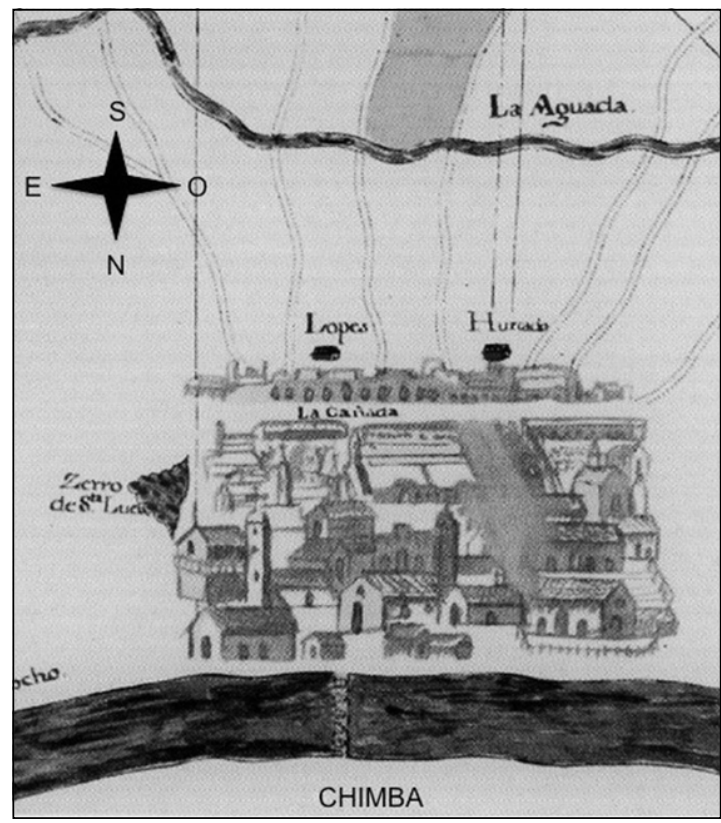

Figura 1. Plano de Santiago de 1772 confeccionado por Antonio Lozada (Archivo Nacional Histórico). Se observa como linde oriente de la ciudad el cerro Santa Lucía, al norte el río Mapocho y al sur la Cañada Al poniente no aparece un límite preciso, pero éste correspondía al deslinde oriente de la chacra de don Diego García de Cáceres (actualmente avenida Brasil). Al sur se observa el trayecto del Zanjón de la Aguada.

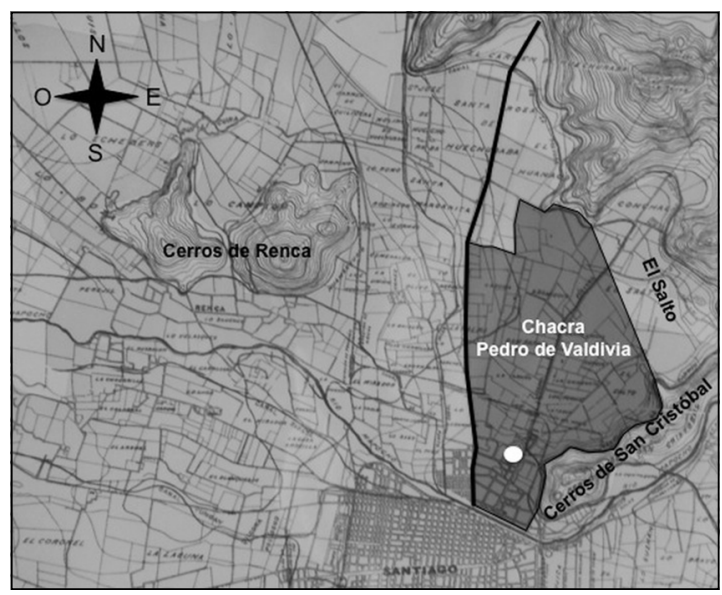

Figura 2. En este esquema se observa en gris la chacra de don Pedro de Valdivia en la Chimba, posteriormente llamada chacra de Montserrat o Llano de Santo Domingo. Se destaca en un círculo blanco el cerro denominado "Cerro Blanco" o "de Montserrat" y en línea negra el antiguo camino real de Huechuraba o de Chile (hoy una sección de la avenida Independencia). Se destacan además los cerros de Renca y San Cristóbal como referencias geográficas. El plano base utilizado para esta figura fue publicado por la Sociedad del Canal del Maipo en 1905 (archivo privado). donó su chacra de La Chimba a su compañera y amiga doña Inés de Suárez, quien después de la muerte del gobernador contrajo matrimonio con don Rodrigo de Quiroga y López de Ulloa, futuro gobernador de Chile en dos períodos (1565-1567 y 1575-1580). Inés de Suárez, devota de la virgen de Montserrat o Patrona de Cataluña, había fundado una ermita o capilla en la cumbre del Cerro Blanco de la Chimba de Santiago en 1545. La donación realizada por Pedro de Valdivia fue en realidad para la institución de la ermita del Cerro Blanco con el objeto que ella pudiera aprovechar sus utilidades, y no como un bien personal para Inés de Suárez. Los patronos de la ermita eran en ese entonces doña Inés y su esposo don Rodrigo de Quiroga. Ambos cónyuges decidieron en 1558 instituir una capellanía (fundación en que ciertos bienes quedan sujetos al cumplimiento de misas u obras pías) en la ermita y su chacra anexa, y entregarla a los padres del convento de Santo Domingo. De esta manera, los padres de Santo Domingo pasaron a ser, con el tiempo y luego de varias disputas, los dueños de la chacra que pasó a denominarse "chacra del Llano de Santo Domingo" o, simplemente, el Llano de Santo Domingo³.

\section{Quinta del catedrático Doctor Ignacio de Jesús Zambrano}

A inicios del s. XVII los padres dominicos vendieron una pequeña superficie de su chacra de Santo Domingo, de aproximadamente $6 \mathrm{Ha}$, a Diego de Aranda Gatica y su esposa Juana de Azócar ${ }^{4}$. Dicho terreno de la Chimba, no diferente a tantos otros de la época, tendría un destino singular, pues sería el origen del emplazamiento del antiguo Hospital San Vicente de Paul y el actual Campus Norte de la Facultad de Medicina de la Universidad de Chile. Dicho terreno que desde ahora denominaremos "quinta", lindaba por la parte del poniente con la Cañadilla y estaba localizado inmediatamente al sur de las tierras del futuro Panteón o cementerio de la ciudad (Figura 3). A mediados del s. XVIII los descendientes de Diego de Aranda vendieron dicha quinta al médico peruano Ignacio de Jesús Zambrano (escritura no encontrada). Paralelamente a estos hechos, se estableció formalmente en 1748 la Real Universidad de San Felipe en Santiago, pero sus clases comenzaron recién una década después, en 1758. 


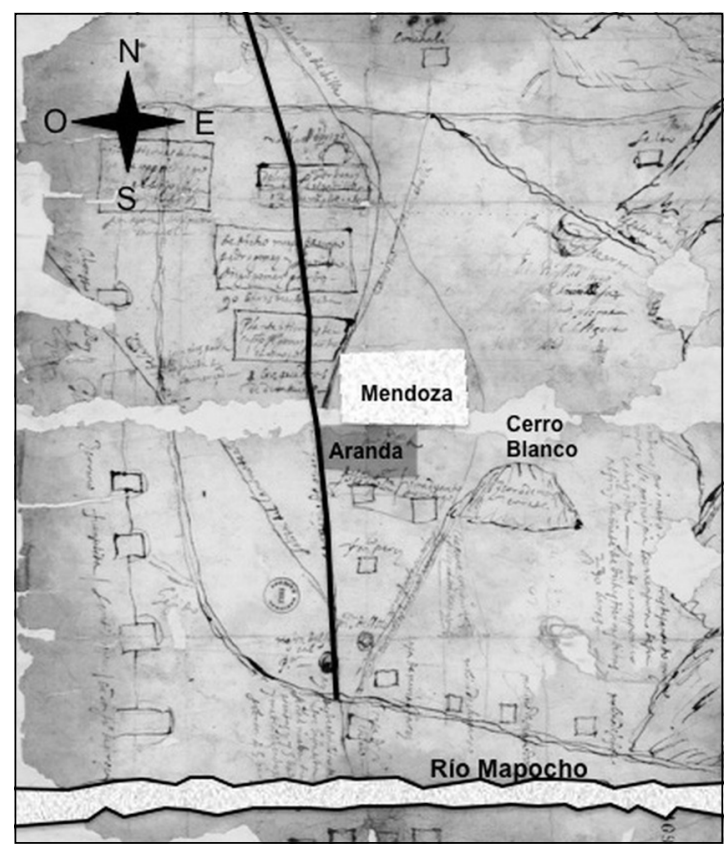

Figura 3. Se muestra una sección de la Chimba del año de 1642 (Archivo Nacional Histórico). Se destaca con un rectángulo plomo la quinta de Diego de Aranda, en blanco las tierras de Álvaro de Mendoza (actualmente Cementerio General) y en línea negra el camino real de Huechuraba (hoy una sección de la avenida Independencia). Además se indica el trayecto del río Mapocho en blanco.

Entre las cátedras que se establecieron en la Real Universidad, la de "Prima Medicina" tiene una especial importancia para este trabajo (su nombre deriva del hecho que se dictaba por la mañana en contraposición con la otra Cátedra de Medicina, llamada "de Vísperas", que se realizaba por la tarde; además existían en otras universidades de la época las Cátedras de Método o Terapéutica y Anatomía o Cirugía; en Chile, por falta de recursos sólo se dictó la de Prima). El primer catedrático de Prima Medicina fue el profesor Doctor Domingo Nevin (1722-1770), nacido en Irlanda y luego titulado en Francia (llegó a Chile en 1754). Por muerte del Dr. Nevin en 1770, se declaró vacante la Cátedra de Prima Medicina, nombrándose primero en calidad de interino y luego como titular al Doctor Ignacio de Jesús Zambrano. El Dr. Zambrano había adquirido su grado de bachiller en Medicina en la Universidad de Lima y posteriormente en 1756 el grado de doctor en la Universidad de San Felipe de Santiago de Chile.
El Dr. Zambrano fue titular de dicha Cátedra de Medicina hasta el año 1786 en que falleció ${ }^{5}$. Seguramente el Dr. Zambrano utilizaba su quinta como un lugar de descanso y sosiego, alejado del mundanal ruido de la ciudad y su continua efervescencia. Es posible además que allí tuviese una vetusta biblioteca con obras de Hipócrates, Galeno y Avicena, padres ilustres de la enseñanza médica y que Zambrano frecuentemente consultase para apoyar sus lecciones en la Universidad. Después de fallecer el Dr. Zambrano, su viuda e hijos decidieron vender la quinta al comerciante español Francisco Javier Matte y Pérez de Ramos, con fecha 5 de julio de $1798^{6}$. Azar, necesidad histórica, jugarreta de la Moiras, el hecho es que un destacado médico y catedrático colonial vino a entreverarse estrechamente con el sino que Asclepio ya había designado a estas tierras, anticipándose en más de una centuria a su incorporación definitiva a la historia médica nacional.

\section{Quinta de don Francisco Javier Matte}

Francisco Javier Matte Pérez fue un comerciante español, oriundo de Santander, que contrajo matrimonio en 1782 con una destacada dama santiaguina llamada María del Rosario Messía Cereceda. A don Francisco Javier le correspondió ser el genearca (fundador de su linaje) de la familia Matte en Chile. Una anécdota cuenta que, después de la batalla de Cancha Rayada (19 de marzo de 1818), don Francisco Javier, al igual que otras personas de fortuna de la época, enterró su oro y joyas en el sitio de una mujer pobre. El estado patriota descubrió finalmente el tesoro, confiscando una parte y devolviendo el resto a su dueño ${ }^{7}$. Algunas de sus hijas heredaron diferentes partes de la quinta de la Cañadilla y las conservaron hasta la década de 1850. Ellas fueron doña Manuela, que contrajo matrimonio con Eduardo Mac Clure Macracken, doña Jesús, casada con José Ignacio de la Plaza Flores y doña Dolores Matte Messía. Posteriormente la sección norte de la quinta fue reunificada por el nieto de don Francisco Javier, el destacado banquero, empresario y filántropo Carlos Mac Clure Matte, mediante dos compras realizadas en la década de $1850-1860^{8}$. La sección sur de la quinta fue adquirida por don Pedro Félix Rodríguez Garzo, en 1871, y luego comprada por el fisco. Los deslindes de la quinta completa 


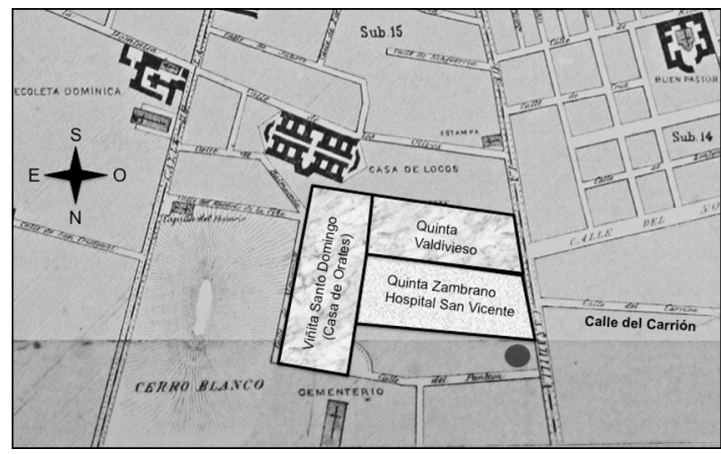

Figura 4. Se muestra la ubicación de la quinta Zambrano y sus principales deslindes; al sur se muestra la quinta que fue de Manuel Joaquín de Valdivieso Maciel (primer administrador del Cementerio General y gestor de la compra de dichos terrenos a los padres de Santo Domingo), al oriente los terrenos de la Viñita de Santo Domingo (adquiridos posteriormente por la Casa de Orates), al poniente la Cañadilla y la calle de Carrión y al norte sitio donde posteriormente se construiría la Escuela de Medicina de la Cañadilla (círculo gris).

(sección norte y sur unidas) eran los siguientes: al poniente, calle Cañadilla por medio con callejón de Carrión; al sur, la quinta Valdivieso; al oriente la casa de Orates y al norte varios vecinos (Figura 4). Es interesante recordar que en la antigua Plaza de Armas de Santiago, en su lado oriente, existió por varias décadas el recordado Portal Mac Clure (hoy Portal Bulnes), perteneciente a este señor. La escritura de donación o venta de la quinta Zambrano al fisco por parte de los herederos de Carlos Mac Clure aún no ha sido encontrada. Sin embargo, un antecedente relevante es que Domingo Matte Messía (1808-1879), tío de Carlos Mac Clure Matte e hijo de Francisco Javier Matte, fue nombrado presidente de la comisión para la construcción de los hospitales del Salvador y San Vicente de Paul'. Es probable que la donación o venta haya sido realizada informalmente por la viuda de Mac Clure a su pariente Domingo Matte. Un signo que refleja esta probable informalidad es que doña Francisca Ossandón Aldunate, esposa y heredera de Carlos Mac Clure, aparece aún como propietaria de la quinta en una escritura fechada en noviembre de 1873, o sea casi un año después de que el Hospital San Vicente comenzara a construirse (primero de septiembre de 1872). De esta forma, el terreno de la quinta del Dr. Zambrano y luego del mercader don Francisco Javier Matte fue destinado a la construcción de un nuevo hospital para enfermedades contagiosas denominado San Vicente de Paul y que junto con el Hospital del Salvador renovarían el par de antiguos hospitales coloniales que Santiago aún conservaba ${ }^{10,11}$. El Hospital San Vicente de Paul fue el precursor histórico directo del actual Hospital Clínico de la Universidad de Chile (antes denominado Hospital José Joaquín Aguirre) y en sus terrenos se construyeron el actual hospital clínico y el edificio del Campus Norte de la Facultad de Medicina de la Universidad de Chile.

\section{Epílogo}

Recientemente, durante la excavación para la futura estación "Hospitales", del Metro de Santiago, fueron descubiertos restos de una quinta ubicada en la esquina sur-oriente de la calle Profesor Zañartu (antigua calle del Panteón) y la avenida Independencia (antigua calle de la Cañadilla). Esta quinta perteneció a la familia Rodríguez Garzo y era una de las cuatro quintas que se localizaban al norte de la quinta del Dr. Zambrano (entre la calle Panteón y la quinta Zambrano, Figura 4). Esta quinta fue posteriormente adquirida por el fisco para construir la entrada y jardines de la antigua Escuela de Medicina de la Cañadilla ${ }^{12}$.

Agradecimientos: A Valeria Sabaj Diez y Javier Osorio Naranjo por sus excelentes sugerencias y correcciones del manuscrito original, al Museo de Historia de la Medicina de la Facultad de Medicina de la Universidad de Chile y al Sr. José Fernández Maturana, de la sección Revistas de la Biblioteca de la Facultad de Medicina de la Universidad de Chile. Además, agradezco al Padre Ramón Ramírez, O. P. encargado del Archivo del Convento de Santo Domingo, y al Archivo Nacional Histórico por permitirme acceder a documentos originales imprescindibles para este estudio.

\section{Referencias}

1. Sotomayor G, Stehberg R. Mapocho Incaico. Boletín Museo Nacional de Historia Natural 2013; 61: 85-149.

2. Almeyda A. Mensuras de Ginés de Lillo. Imprenta Universitaria. Santiago de Chile, 1942.

3. Ghigliazza R. Historia de la Provincia Dominicana de Chile. Tomo I. Santiago de Chile, 1898. 
4. Archivo Convento Santo Domingo. Escritura de censo o hipoteca de Diego de Aranda y su esposa Juana de Azócar sobre su Quinta de la Cañadilla a favor del Convento de Santo Domingo fechada en el año 1626 ante el escribano Juan Donoso Pajuelo. Tomo D2-20. Biblioteca Convento de Santo Domingo, Santiago, Chile.

5. Medina JT. Historia de la Real Universidad de San Felipe. Imprenta Universo. Santiago de Chile, 1928.

6. Fondo Escribanos Santiago. Tomo 858 de Francisco Borja de la Torre. Fecha: 1796-1799.

7. Salazar G. Mercaderes, empresarios y capitalistas. $2^{\circ}$ edición, editorial Sudamericana, Santiago de Chile, 2011.

8. Conservador de Bienes Raíces de Santiago (CBRS). Escrituras de compraventa citadas en el texto: Compraventa de la sección norte de la quinta por Carlos Mac Clure a su tía Dolores Matte Messía y otra a su hermano
Ignacio Mac Clure (registros No 214 y 215 de 1869, respectivamente). Compra de sección sur por Pedro Félix Rodríguez Garzo a Jesús Matte en 1871 (registros No 967 de 1871). Venta de Pedro Rodríguez Garzo al Hospital San Vicente (registro No 260 de 1882). Adjudicación de la quinta a Francisca Ossandón Aldunate (registro No 863 de 1873).

9. Rosales J. La Cañadilla de Santiago: su Historia y sus Tradiciones 1541-1887. Sangría Editora, Santiago, Chile, 2010.

10. Cruz-Coke R. Historia de la Medicina Chilena. Editorial Andrés Bello, primera Edición, Santiago de Chile, 1995.

11. Osorio CG. Arqueología del Hospital San Vicente de Paul. Rev Med Chile 2007; 135: 264-9.

12. Osorio, CG. Escuela Medicina de la Cañadilla. Rev Med Chile 2013; 11: 1484-8. 